\title{
Sympathetic activity-associated periodic repolarization dynamics predict mortality following myocardial infarction
}

Konstantinos D. Rizas, ${ }^{1}$ Tuomo Nieminen, ${ }^{2}$ Petra Barthel, ${ }^{3}$ Christine S. Zürn, ${ }^{1}$ Mika Kähönen, ${ }^{4}$ Jari Viik, ${ }^{5}$ Terho Lehtimäki, ${ }^{6}$ Kjell Nikus, ${ }^{7}$ Christian Eick, ${ }^{1}$ Tim O. Greiner, ${ }^{8}$ Hans P. Wendel, ${ }^{8}$ Peter Seizer, ${ }^{1}$ Jürgen Schreieck, ${ }^{1}$ Meinrad Gawaz, ${ }^{1}$ Georg Schmidt, ${ }^{3}$ and Axel Bauer ${ }^{1}$

\author{
${ }^{1}$ Medizinische Klinik III, Abteilung für Kardiologie und Herz-Kreislauferkrankungen, Eberhard Karls University, Tübingen, Germany \\ ${ }^{2}$ Division of Cardiology, Helsinki University Central Hospital, Helsinki, Finland. ${ }^{31}$. Medizinische Klinik, Technische Universität München, Munich, Germany. \\ 4Department of Clinical Physiology and 5Department of Biomedical Engineering, Tampere University of Technology, Tampere, Finland. \\ ${ }^{6}$ Department of Clinical Chemistry, Fimlab Laboratories, School of Medicine at University of Tampere, Tampere, Finland. \\ ${ }^{7}$ Heart Centre, Department of Cardiology, Tampere University Hospital, Tampere, Finland. \\ ${ }^{8}$ Department of Thoracic, Cardiac and Vascular Surgery, Eberhard Karls University, Tübingen, Germany.
}

\begin{abstract}
Background. Enhanced sympathetic activity at the ventricular myocardium can destabilize repolarization, increasing the risk of death. Sympathetic activity is known to cluster in low-frequency bursts; therefore, we hypothesized that sympathetic activity induces periodic low-frequency changes of repolarization. We developed a technique to assess the sympathetic effect on repolarization and identified periodic components in the low-frequency spectral range $(\leq 0.1 \mathrm{~Hz})$, which we termed periodic repolarization dynamics $(P R D)$.
\end{abstract}

Methods. We investigated the physiological properties of PRD in multiple experimental studies, including a swine model of steady-state ventilation $(n=7)$ and human studies involving fixed atrial pacing $(n=10)$, passive head-up tilt testing $(n=11)$, low-intensity exercise testing $(n=11)$, and beta blockade $(n=10)$. We tested the prognostic power of PRD in 908 survivors of acute myocardial infarction (MI). Finally, we tested the predictive values of PRD and T-wave alternans (TWA) in 2,965 patients undergoing clinically indicated exercise testing.

Results. PRD was not related to underlying respiratory activity $(P<0.001)$ or heart-rate variability $(P=0.002)$. Furthermore, PRD was enhanced by activation of the sympathetic nervous system, and pharmacological blockade of sympathetic nervous system activity suppressed PRD $(P \leq 0.005$ for both). Increased PRD was the strongest single risk predictor of 5-year total mortality (hazard ratio $4.75,95 \%$ CI $2.94-7.66 ; P<0.001$ ) after acute MI. In patients undergoing exercise testing, the predictive value of PRD was strong and complementary to that of TWA.

Conclusion. We have described and identified low-frequency rhythmic modulations of repolarization that are associated with sympathetic activity. Increased PRD can be used as a predictor of mortality in survivors of acute $\mathrm{MI}$ and patients undergoing exercise testing.

Trial registration. ClinicalTrials.gov NCT00196274.

Funding. This study was funded by Angewandte Klinische Forschung, University of Tübingen (252-1-0).

\section{Introduction}

Sudden cardiac death (SCD) is the single most common cause of death in the industrialized world (1). A substantial proportion of SCD cases occur in patients after myocardial infarction (MI). Randomized trials have demonstrated that in high-risk patients after MI, mortality can be effectively reduced by prophylactic implantation of a cardioverter-defibrillator (ICD) (2). Consequently, identification of high-risk individuals is a major objective in cardiology. Current guidelines recommend the assessment of left ventricular ejection fraction (LVEF) as the gold standard risk predictor $(3,4)$; however, this approach lacks both sensitivity and specificity $(1,5)$. Therefore, development of novel risk markers is of great clinical interest.

Conflict of interest: The authors have declared that no conflict of interest exists. Citation for this article: J Clin Invest. 2014;124(4):1770-1780. doi:10.1172/JCI70085
Assessment of repolarization instability may more directly estimate the risk of fatal cardiac arrhythmias (6). It is well known from experimental and clinical studies that enhanced sympathetic activity is a key factor leading to the destabilization of myocardial repolarization (7-14). However, without directly recording neural activity, which is impractical in the clinical setting, assessment of the sympathetic effect on myocardial repolarization has not been possible to date. As sympathetic activity is organized in a series of low-frequency bursts (15-19), we postulated that repolarization changes induced by the sympathetic nervous system would exhibit low-frequency periodic features.

In the present study, we propose what we believe is a novel way to assess the sympathetic effect on cardiac repolarization. We developed a technology and uncovered periodic components of repolarization in the low-frequency spectral range $(\leq 0.1 \mathrm{~Hz})$, which we termed periodic repolarization dynamics (PRD). The first part of 

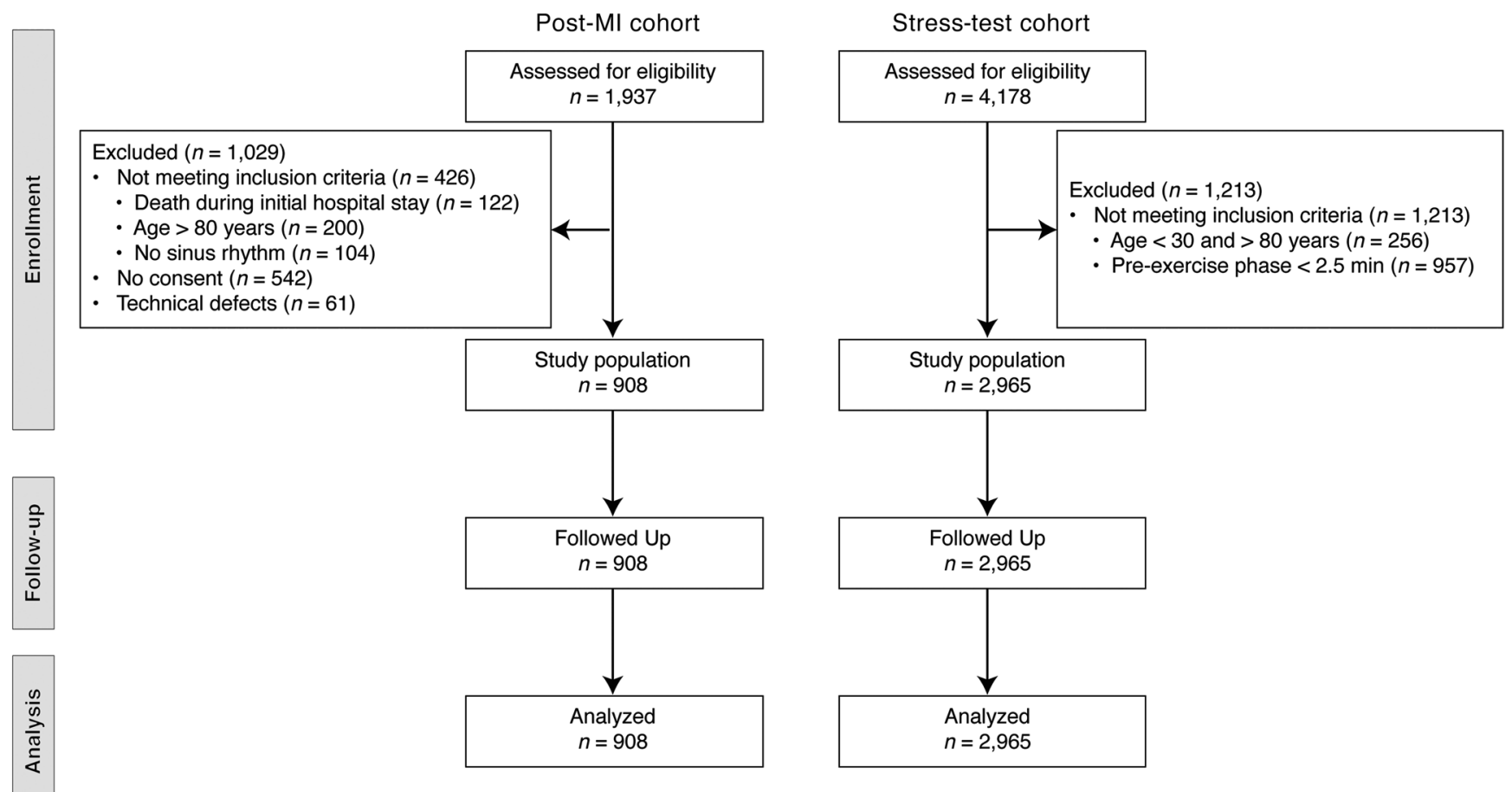

Figure 1

CONSORT flow diagrams. Enrollment, follow-up, and analysis in the post-MI and stress-test cohorts.

this article focuses on the physiological properties of PRD, including activation and blockade of the sympathetic nervous system. In the second part of this investigation, we assess the prognostic meaning of enhanced PRD in patients surviving acute MI (postMI cohort; Figure 1A) and patients undergoing clinically indicated exercise testing (stress-test cohort; Figure 1B). In the stress-test cohort we also tested the prognostic meaning of exercise-induced T-wave alternans (TWA), which is presently considered to be the strongest existing marker of repolarization instability.

\section{Results}

Repolarization is subject to low-frequency periodic modulations. We developed a technique to dynamically track repolarization dynamics and to quantify their periodic components. Details of the methodology are reported in Methods. Briefly, we used standard, high-resolution, surface ECG recorded in or converted to the orthogonal Frank lead configuration. As electrocardiographic repolarization is a phenomenon occurring in both space and time, we integrated the spatiotemporal information of each $\mathrm{T}$-wave into a single vector, $T^{\circ}$. We used the angle $d T^{\circ}$ between successive repolarization vectors as an estimate of the instantaneous repolarization instability (Figure 2, A-C). We observed characteristic low-frequency oscillations in $d T^{\circ}$ in health and disease (Figure $2 \mathrm{D}$ ). In order to quantify these low-frequency $(\leq 0.1 \mathrm{~Hz})$ periodic patterns, we employed wavelet analysis (Figure 2E).

$P R D$ is not an epiphenomenon of underlying heart rate variability. We tested whether PRD was present in the absence of heart rate variability (HRV). We studied 10 individuals (median age 52 [interquartile range (IQR) 32] years, 5 females), who underwent a clinically indicated electrophysiological (EP) study at our institution. Patient characteristics are provided in Methods. We compared 5-minute episodes of spontaneous sinus rhythm to 5-minute episodes during fixed atrial stimulation, which was set above the spontaneous heart rate. Fixed atrial pacing almost abolished HRV $(P<0.001$; Supplemental Table 1; supplemental material available online with this article; doi:10.1172/JCI70085DS1), but exerted only minimal, nonsignificant effects on PRD (ratio of PRD after provocation to PRD before provocation [PRD ratio] 0.75, 95\% CI 0.50-1.17, $P=0.193$; Figure 3A, Supplemental Figure 1A, and Supplemental Table 1).

$P R D$ is not an epiphenomenon of underlying respiratory activity. To test whether PRD was present in the absence of spontaneous breathing, we performed an experimental study in a swine model. Seven female domestic pigs were mechanically ventilated and sedated with $\alpha$-chloralose, which has been shown to induce only minimal effects on the cardiac autonomic nervous system (20). Respiratory frequency and tidal volume were maintained constant by means of volume-controlled ventilation. Details of the experimental design are provided in Methods. PRD occurred independently of respiratory activity, as illustrated in Figure 4A. There was no interference between respiratory activity and PRD in any animal, as confirmed by spectral and crossspectral analysis (Figure 4, B and C; median coherence 0.044 [IQR 0.026]; $P<0.001$ for the difference from the threshold of 0.5).

$P R D$ is enhanced by sympathetic activation and suppressed by sympathetic blockade. We tested the effects of sympathetic activation on PRD in 11 healthy male volunteers (median age 24 [IQR 3] years). Sympathetic activation was achieved by means of head-up tilt testing and low-intensity exercise. Both tilt-table testing (PRD ratio 1.80, 95\% CI 1.35-2.58, $P=0.005$; Figure 3B, Supplemental Figure 1B, and Supplemental Table 1) and low-intensity exercise (PRD ratio 3.85, 95\% CI 2.49-5.61, $P=0.001$; Figure 3C, Supplemental Figure 1B, and Supplemental Table 1) led to substantial enhancement of PRD. 
clinical medicine

A

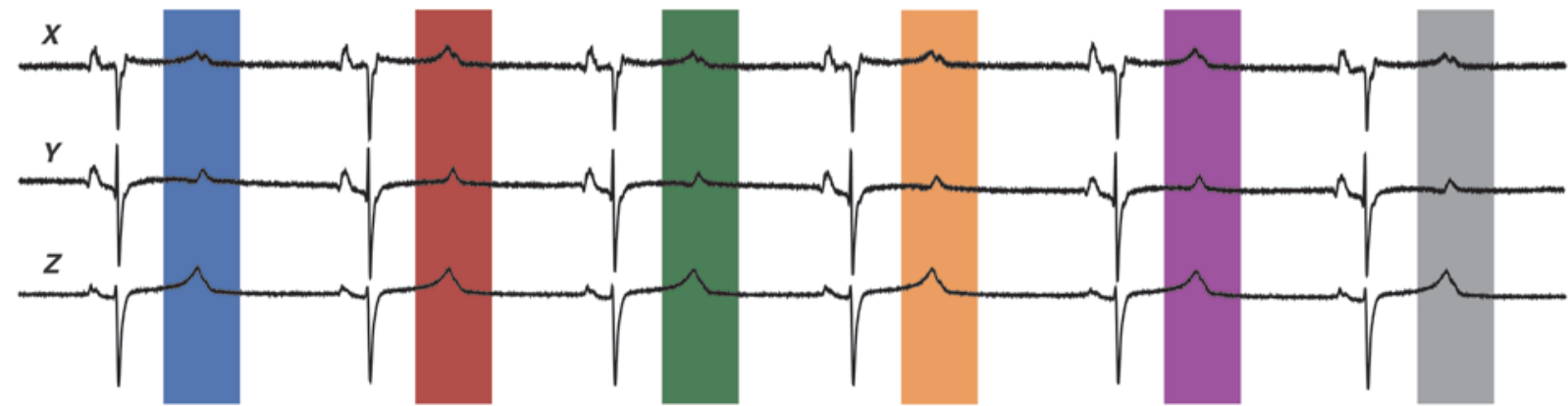

B
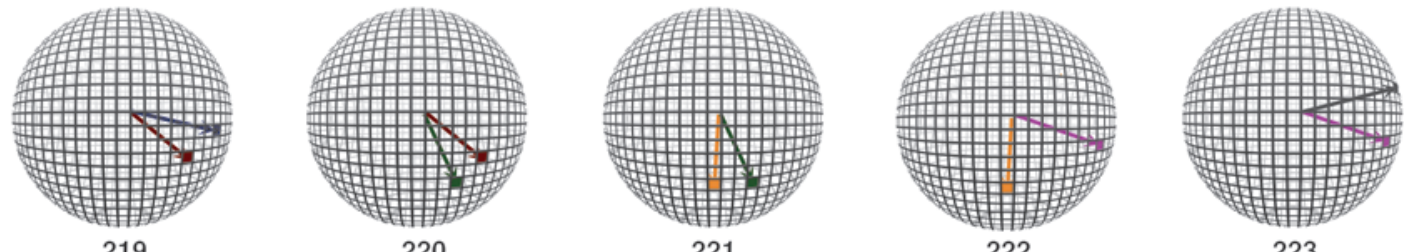

C

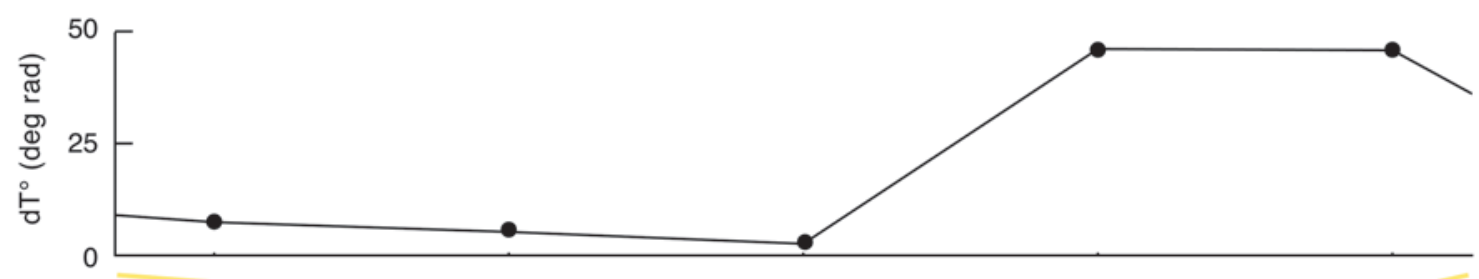

D

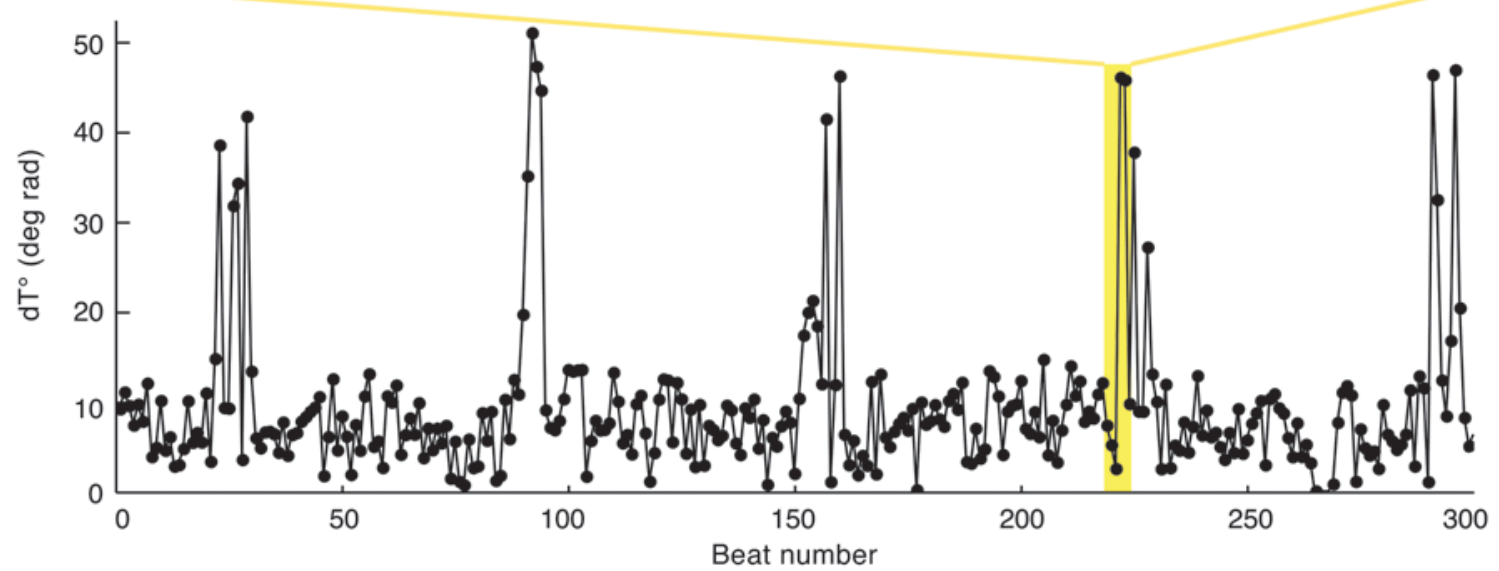

E

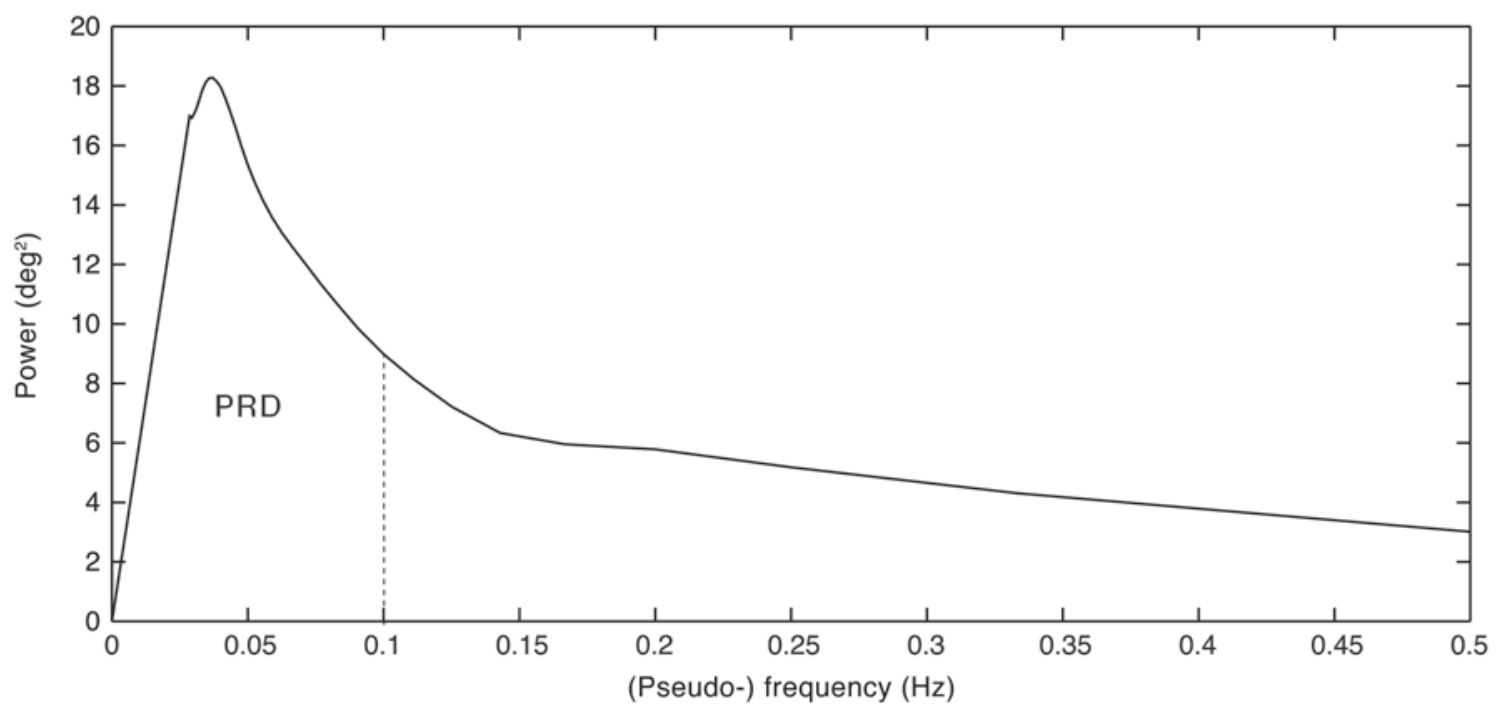




\section{Figure 2}

Assessment of PRD. (A) Illustration of the weight-averaged vector of repolarization $\left(T^{\circ}\right)$ for each T-wave from surface ECG recorded in the Frank leads configuration. (B) Three-dimensional visualization of successive $T^{\circ}$ vectors projected into virtual spheres. The angle $d T^{\circ}$ between successive repolarization vectors was used as an estimate of instantaneous repolarization instability. (C and D) The $d T^{\circ}$ signal exhibits characteristic low-frequency oscillations. C shows $d T^{\circ}$ values for beats \#219-223, corresponding to the spheres in B. (E) Quantification of PRD using wavelet analysis. PRD was defined as the average wavelet coefficient corresponding to frequencies of $0.1 \mathrm{~Hz}$ or less.

Conversely, we tested the effects of antiadrenergic intervention in 10 patients (median age 57 [IQR 21] years, 7 females) undergoing an EP study at our institution. Antiadrenergic intervention was achieved by pharmacological beta blockade. The diagnostic protocol is described in Methods. Beta blockade caused a striking suppression of PRD in all patients (PRD ratio $0.41,95 \% \mathrm{CI}$ $0.28-0.61, P=0.002$; Figure $3 \mathrm{D}$, Supplemental Figure $1 \mathrm{C}$, and Supplemental Table 1).

For comparison, the effects of sympathetic activation and blockade on the low-frequency component of heart-rate variability are shown in Supplemental Table 1.

Increased PRD predicts total and cardiovascular mortality after MI. We tested the prognostic significance of PRD in a cohort of 908 patients from the Autonomic Regulation Trial (median age 61 [IQR 17] years, 174 females) who survived an acute MI (Figure $1 \mathrm{~A}$ and Table 1) (21, 22). Sixty-nine patients died within the first 5 years of follow-up. Representative resting $d T^{\circ}$ signals in a patient who survived the follow-up period and in a patient who suddenly died 8 months after index MI are depicted in Figure 5, A and B, respectively. Although low-frequency oscillations in $d T^{\circ}$ were evident in both patients, the amplitudes of PRD were much higher in the nonsurviving patient. The level of PRD was significantly associated with 5-year mortality (6.67 [IQR 8.58] deg² vs. 2.66
[IQR 3.93] $\left.\mathrm{deg}^{2} ; P<0.001\right)$. For subsequent survival analyses, we dichotomized PRD at the upper quartile of the study population. The 227 patients with PRD greater than or equal to $5.75 \mathrm{deg}^{2}$ (Figure 5 C) had a 5 -year risk of death of $18.2 \%$ compared with $4.1 \%$ in the 681 patients with PRD of less than $5.75 \mathrm{deg}^{2}(P<0.001)$. Both uni- and multivariable analyses for the prediction of 5-year total mortality indicated that PRD greater than or equal to $5.75 \mathrm{deg}^{2}$ was the strongest single risk predictor in the study cohort (Table 2 and Supplemental Figure 2). The predictive value of PRD greater than or equal to $5.75 \mathrm{deg}^{2}$ was independent of that of established risk markers, including reduced LVEF of $35 \%$ or less $(3,4)$, the Global Registry of Acute Coronary Events (GRACE) score (23), the presence of diabetes mellitus, elevated mean heart rate, reduced HRV, and increased QT variability index (QTVI) (24). Subsequently, we assessed the incremental prognostic value of PRD to established risk-prediction models (Supplemental Table 2). PRD significantly improved all tested risk-prediction models based on the combination of LVEF, GRACE score, respiratory rate, and HRV parameters. Subgroup analyses revealed that increased PRD was a particularly strong predictor of mortality in the 179 post-MI patients who also suffered from diabetes mellitus, identifying a group of 179 patients with a cumulative 5 -year mortality rate of $38.8 \%$ (Figure 5D).

Finally, we also investigated whether PRD predicted cardiovascular mortality. Of the 69 deaths, 36 were cardiovascular deaths. As shown in Table 2, PRD greater than or equal to $5.75 \mathrm{deg}^{2}$ was also a strong and independent predictor of 5-year cardiovascular mortality.

The predictive value of $P R D$ is complementary to that of exercise-induced TWA. To test the predictive values of PRD and TWA, we studied 2,965 patients (median age 57 [IQR 16] years, 1,187 females) from the Finnish Cardiovascular Study (median age 57 [IQR 16], 1,187 females; Figure 1B and Table 1) who underwent a clinically indicated exercise test (25). During a median follow-up of 6 years, 309 patients died. In all patients, TWA was measured during exercise by the modified moving average (MMA) method (25-27). PRD was assessed in the preexercise period with patients sitting on a bicy-
Provocation

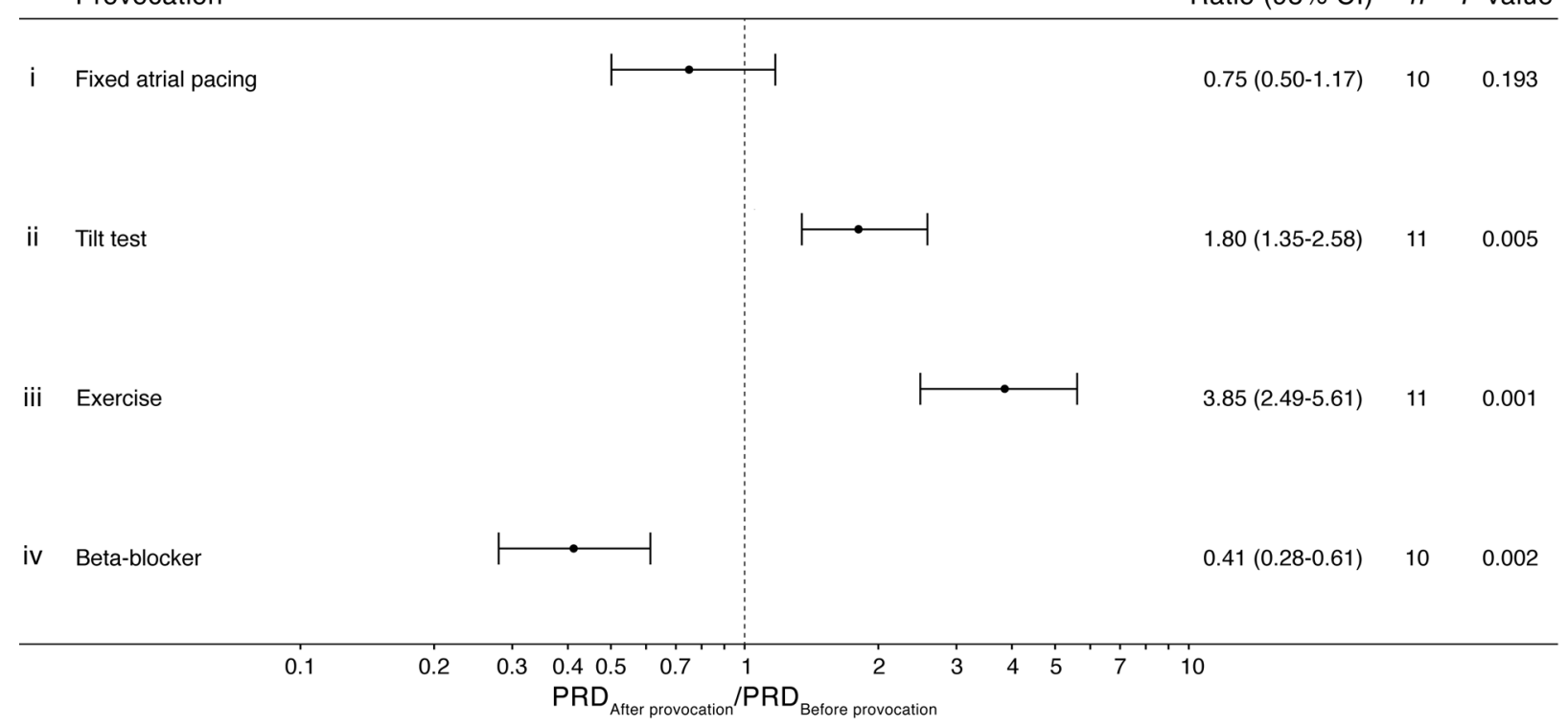

\section{Figure 3}

Physiological and pharmacological provocations. Effects of fixed atrial stimulation (i), tilt-table testing (ii), exercise (iii), and pharmacological beta blockade (iv) on PRD. PRD ratio was plotted on a logarithmic axis and used to quantify the effect of each procedure. 

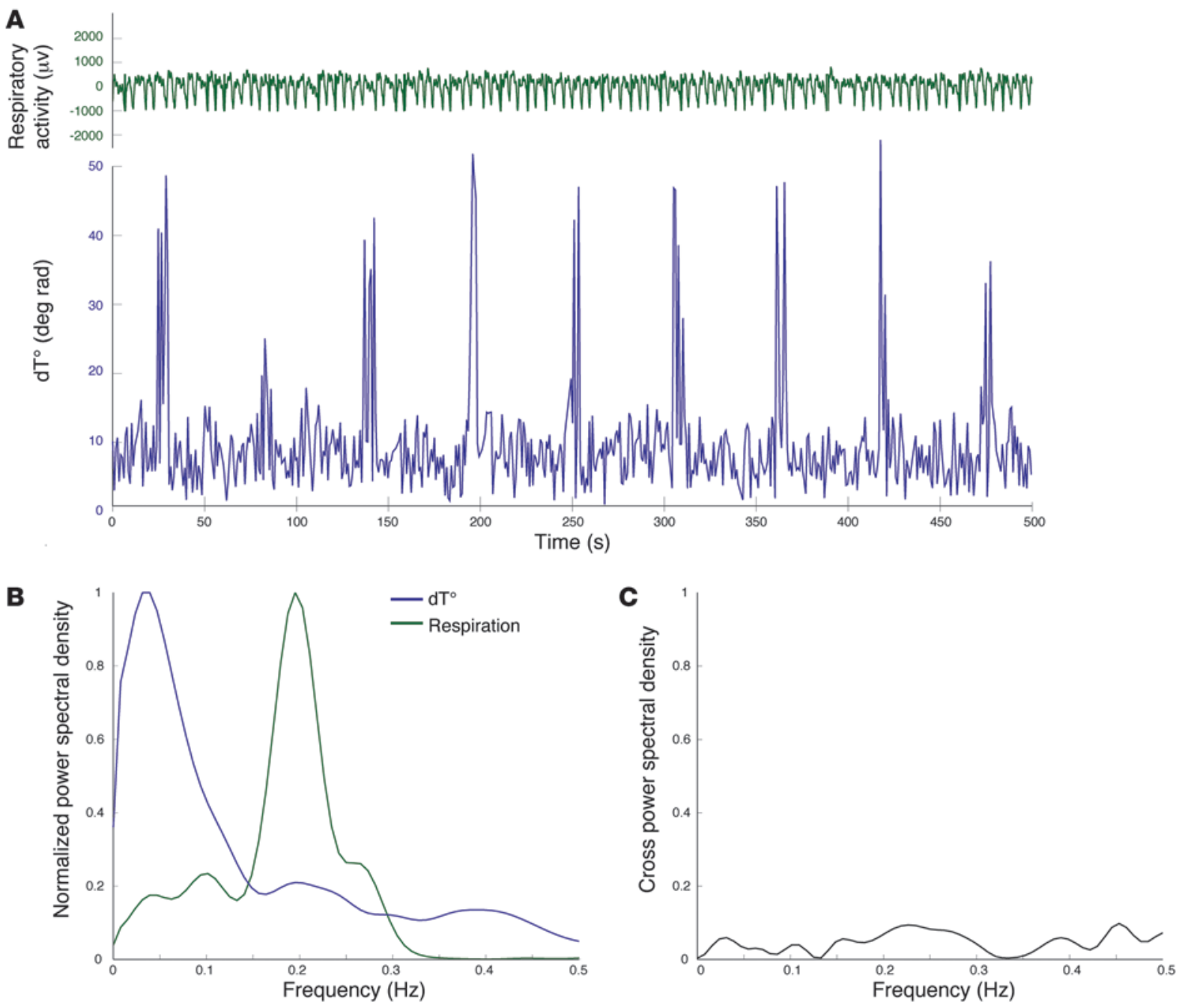

\section{Figure 4}

Effect of respiration on PRD in a volume-controlled ventilated swine. (A) Signals of respiratory activity (green) and $d T^{\circ}$ (blue). Respiratory activity was recorded by a piezoelectric thoracic sensor. The $d T^{\circ}$ signal exhibits typical low-frequency oscillations occurring independently from respiratory activity. (B) Spectral analysis of respiratory activity and the $d T^{\circ}$ signal. Power spectra were normalized by their maximum value. (C) Crossspectral analysis of respiratory activity and the $d T^{\circ}$ signal showing a lack of interference between both signals.

cle ergometer. As expected, PRD levels in this cohort were higher than those in the post-MI cohort, where PRD was estimated in the supine resting position (9.2 [IQR 13.37] $\mathrm{deg}^{2}$ vs. 2.82 [IQR 4.34] $\mathrm{deg}^{2}$, respectively; $\left.P<0.001\right)$. Both PRD and TWA were significantly associated with mortality (8.96 [IQR 13.23] $\mathrm{deg}^{2}$ vs. 12.04 [IQR 16.32] $\mathrm{deg}^{2}, P<0.001$, and 23.00 [IQR 15] $\mu \mathrm{V}$ vs. 26.00 [IQR 17] $\mu \mathrm{V}$, $P<0.001$, respectively). Univariable Cox regression analysis showed that both markers were strong predictors of total mortality (standardized coefficients 0.203 , 95\% CI 0.113-0.293, $P<0.001$ for PRD; and $0.256,95 \%$ CI $0.164-0.348, P<0.001$ for TWA). This remained true on multivariable analysis, which also included age, sex, previous MI, presence of diabetes mellitus, and treatment with beta-blockers (Table 3). The significant crossterm between TWA and PRD (TWA $\times$ PRD) indicated that the relationship between the outcome and one predictor was dependent on the levels of the other predictor. We therefore tested the additive prognostic value of PRD for different levels of TWA. As illustrated in Supplemental Figure 3, PRD provided incremental prognostic information at all levels of TWA.
Of the 309 deaths, 138 were cardiovascular deaths. Increased preexercise PRD was also a significant predictor of cardiovascular mortality in univariable (standardized coefficient $0.256,95 \%$ CI $0.126-0.385 ; P<0.001$ ) and multivariable analysis (Table 3 ).

\section{Discussion}

In the present study, we identified periodic oscillations of repolarization that were localized in the low-frequency spectral range and were detectable by conventional surface ECG. PRD was evident in health and disease without provocations and occurred autonomously from underlying HRV and respiratory activity. PRD was augmented by physiological provocations leading to activation of the sympathetic nervous system and was suppressed by pharmacological adrenergic blockade. Increased PRD obtained under resting conditions was a very strong predictor of total and cardiovascular mortality in survivors of acute MI and patients undergoing a clinically indicated exercise test. The prognostic value of PRD was incremental to that of established risk markers, including LVEF and TWA. 
Table 1

Patient characteristics and treatment in the post-MI and stress-test cohorts

Post-MI cohort

Study characteristics
Number of patients, $n$
Median follow-up (IQR), months
Total deaths, $n(\%)$
Cardiovascular deaths, $n(\%)$
Patient characteristics
Median age (IQR), years
Females, $n(\%)$
Diabetes mellitus, $n(\%)$
History of previous MI, $n(\%)$
Median LVEF (IQR), $\%$
Known CAD (\%)
Treatment

$\mathrm{PCl}, n(\%)$

Thrombolysis, $n(\%)$

CABG, $n(\%)$
Beta blockers, $n(\%)$

\section{Stress-test cohort}

908
$60(0)$
$69(7.6)$
$36(4.0)$

$61(17)$
$174(19.2)$
$179(19.7)$
$86(9.5)$
$53(15)$
$908(100)$

$848(93.4)$

$13(1.4)$

$6(0.7)$

$864(95.1)$

2,965

$75.1(47.7)$

309 (10.4)

$138(4.7)$

57 (16)

$1187(40.0)$

$344(11.6)$

$552(18.6)$

66 (16)

$883(29.8)$
CABG, coronary artery bypass graft; CAD, coronary artery disease; NA, not available; $\mathrm{PCl}$, percutaneous coronary intervention.

ade. Moreover, increased PRD was a strong predictor of total and cardiovascular mortality, which is in line with the results of many studies showing that enhanced sympathetic activity is associated with an increased risk of death (11-14).

In particular, increased PRD was identified as the strongest single risk predictor of total and cardiovascular mortality in a large cohort of post-MI patients. The predictive value of PRD was independent of established risk markers. PRD substantially improved several multivariable models in prediction of total mortality, confirming its incremental prognostic value. The mechanism by which PRD identifies high-risk patients significantly differs from that of structural markers such as LVEF. Directly estimating sympathetic activity at the level of myocardial repolarization may provide more accurate information on cardiac risk. Post-MI patients with increased PRD had a very poor prognosis when they also suffered from diabetes mellitus. Both MI (32) and diabetes mellitus (33) are characterized by spatially heterogeneous sympathetic innervation, which is associated with negative prognosis (10).

We tested the prognostic significance of PRD in a large cohort of patients undergoing clinically indicated exercise testing. Increased PRD was a strong predic-

Noninvasive assessment of the sympathetic effect on myocardial repolarization is of great clinical interest. A wealth of evidence supports the widely held belief that increased sympathetic nervous system activity is associated with increased cardiac vulnerability (11-14). In human subjects, noninvasively measured parameters, including HRV and baroreflex sensitivity, have been employed to study sympathetic activity under routine clinical conditions (28). This approach is based on the principle that activation of the sympathetic nervous system evokes several physiological effects, including increasing systolic contractility rate and vasomotor tone as well as accelerating heart rate and atrioventricular conduction (10). However, these measurements provide only an indirect probe of the sympathetic effect on repolarization; they reflect influences on the sinoatrial node and blood vessels, not on the ventricular myocardium.

At the level of cardiomyocytes, stimulation of $\beta$-adrenergic receptors alters intracellular calcium dynamics (28) and shortens action potential duration (10). Importantly, the effect of sympathetic stimulation on the 3 cell types of the ventricular myocardium (epicardial cells, M cells, and endocardial cells) is nonuniform $(29,30)$. Adrenergic activation abbreviates the action potential duration of epicardial and endocardial cells to a greater degree than the action potential duration of $\mathrm{M}$ cells (31), leading to an increased transmural dispersion of repolarization (28).

It is well known that sympathetic activity is organized in series of low-frequency bursts (15-19). We therefore assumed that phasic sympathetic activation induces phasic changes in repolarization localized in the low-frequency spectral range, and we developed a method to track the sympathetic effect on transmural dispersion of repolarization. Our findings confirmed this hypothesis. For what we believe is the first time, we detected periodic changes in repolarization in the same range as those of the sympathetic nervous system. PRD was significantly enhanced by sympathetic activation and was substantially suppressed by sympathetic block- tor of total and cardiovascular mortality and provided incremental prognostic information to that of exercise-induced TWA. This indicates that PRD can be used to detect high-risk patients who are not identified by TWA. The complementary prognostic information provided by PRD and TWA implies that these 2 markers capture different aspects of repolarization instability. While PRD most probably reflects low-frequency oscillations related to sympathetic activity, TWA is mainly caused by high-frequency action potential oscillations provoked by abnormal calcium handling (34). TWA is an important predictor of cardiovascular mortality, including sudden death $(6,27,35,36)$. However, it needs to be provoked by exercise (37) or invasive procedures (38). Assessment of PRD is inexpensive, easily obtainable under resting conditions, and noninvasive and significantly improves available risk-stratification strategies.

Our study has several limitations. First, high-resolution ECG is required in order to measure PRD. It remains to be demonstrated whether our results are reproducible with lower-resolution tracings. Second, in both cohorts, risk markers were only assessed at enrollment. Therefore, we cannot comment on the immediate and long-term reproducibility of PRD as well as the effect of treatment on PRD. Third, the prognostic value of PRD needs to be validated in independent cohorts. Fourth, as PRD is dependent on the patient's body position and activity level, the proposed cutoff value is only valid for recordings obtained in the supine resting position. Fifth, we confirmed the prognostic value of PRD for prediction of total mortality and cardiovascular mortality. Although it is plausible to assume that increased levels of PRD are also associated with arrhythmic mortality, this needs to be tested in future studies. Finally, although we have shown that increased PRD is a powerful risk predictor, we have no data to show that specific treatments based on the use of this predictor will improve patient outcome.

In conclusion, PRD constitutes an electrocardiographic phenomenon that most likely reflects the myocardial response to sympathetic activation. Increased PRD is a potent risk predictor 
A



C

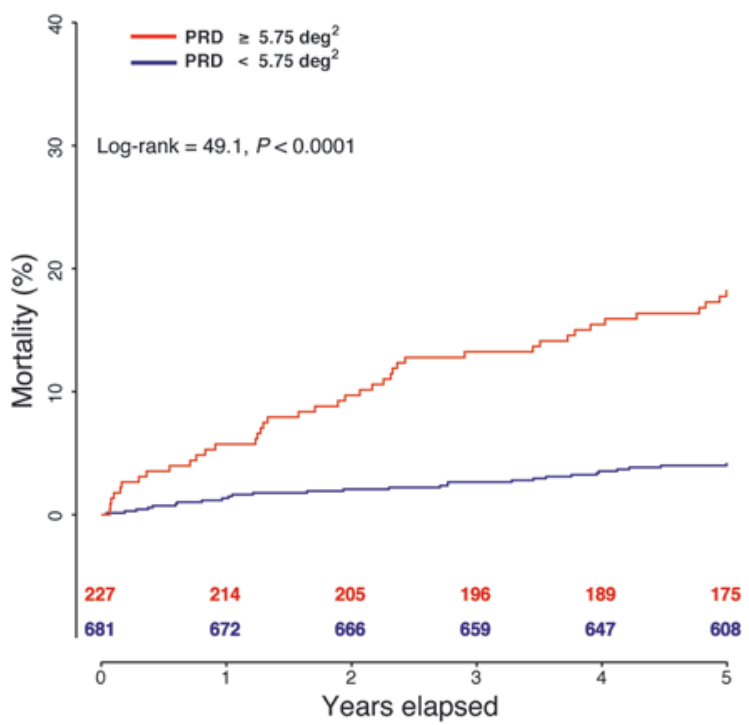

B

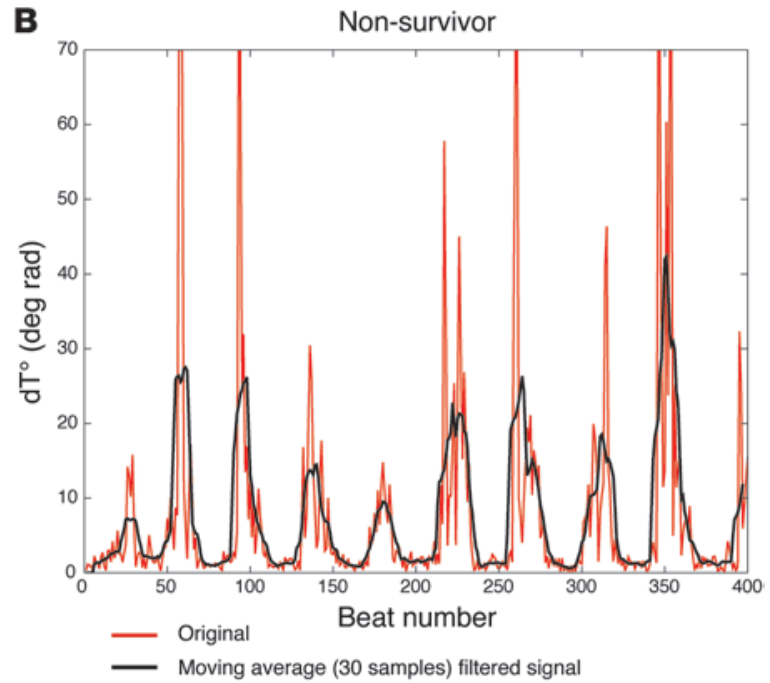

D

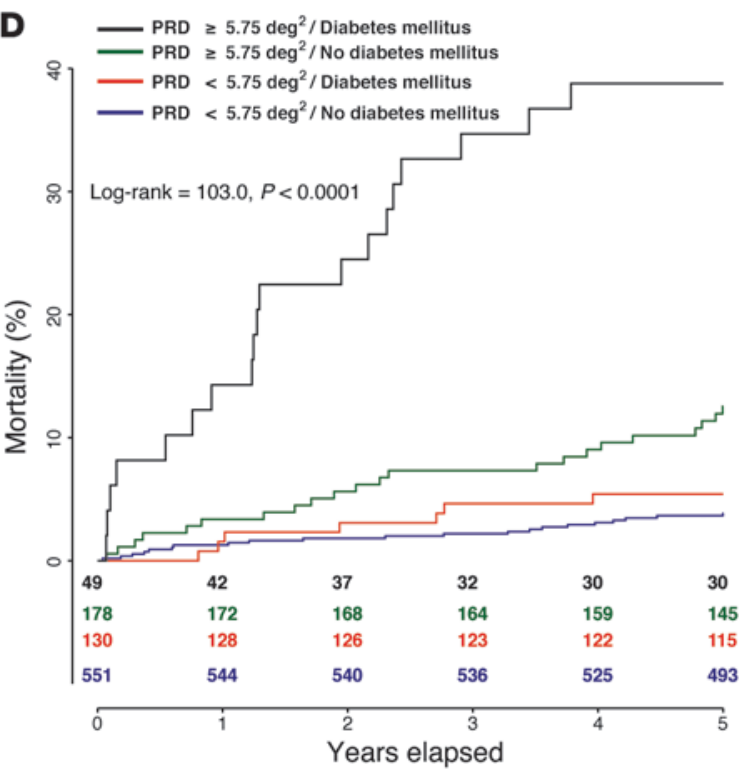

Figure 5

PRD in post-MI patients. (A) Typical $d T^{\circ}$ signal (blue line) obtained from a 50-year-old post-MI patient who survived the 5-year follow-up period. The signal shows characteristic low-frequency oscillations. For better illustration of these oscillations, a low-pass filter was applied and plotted on top of the original signal (black line). (B) Typical $d T^{\circ}$ signal (red line) from a 75-year-old post-MI patient who suddenly died 8 months after MI. Compared with the survivor, the amplitude of PRD was substantially enhanced. (C) Cumulative mortality rates of patients stratified by PRD of $5.75 \mathrm{deg}^{2}$ or more. (D) Cumulative mortality rates of patients stratified by PRD of $5.75 \mathrm{deg}^{2}$ or more and presence of diabetes mellitus.

of total and cardiovascular mortality, and its use significantly improves established risk-stratification concepts. Future studies are needed to test whether high-risk patients identified by PRD benefit from prophylactic therapies.

\section{Methods}

Participants. The physiological properties of PRD were studied in 3 cohorts at the University Hospital of Tübingen. We tested the effects of fixed atrial pacing (atrial-pacing cohort) in 10 individuals (median age 52 [IQR 42] years, 5 females) undergoing a clinically indicated diagnostic EP study. Indications for EP studies were paroxysmal supraventricular tachycardia in 7 patients and evaluation of unexplained syncope in 3 patients.
We investigated the effect of beta blockade in 10 patients (median age 57 [IQR 21] years, 7 females) undergoing an EP study for paroxysmal supraventricular tachycardia (adrenergic-blockade cohort). In both EP studies, all patients were in sinus rhythm, had normal LVEF, were not suspected of suffering from coronary artery disease, and had no significant valve stenosis or insufficiency on echocardiography. We also studied the effects of passive head-up tilt and low-intensity exercise in 11 healthy male volunteers (median age 24 [IQR 3] years, adrenergic-activation cohort).

The prognostic power of PRD was tested in 908 survivors (median age 61 [IQR 17] years, 174 females) of acute MI (post-MI cohort; Figure 1A and Table 1) and 2,965 patients (median age 57 [IQR 16] years, 1,187 females) undergoing clinically indicated exercise testing (stress-test cohort; Figure 


\section{Table 2}

Univariable and multivariable association of risk markers with 5-year all-cause and cardiovascular mortality in 908 survivors of acute MI (post-MI cohort)

\begin{tabular}{|c|c|c|c|c|}
\hline \multirow{3}{*}{ Risk variable } & \multicolumn{4}{|c|}{ All-cause mortality } \\
\hline & \multicolumn{2}{|c|}{ Univariable Cox regression } & \multicolumn{2}{|c|}{ Multivariable Cox regression } \\
\hline & Hazard ratio $(95 \% \mathrm{Cl})$ & $P$ value & Hazard ratio $(95 \% \mathrm{Cl})$ & $P$ value \\
\hline LVEF $\leq 35 \%$ & 3.81 (2.23 to 6.51$)$ & $<0.001$ & $2.13(1.22$ to 3.70$)$ & 0.008 \\
\hline GRACEA $^{A}$ score $\geq 120$ & 5.54 (3.24 to 9.46$)$ & $<0.001$ & 3.61 (2.06 to 6.31$)$ & $<0.001$ \\
\hline Diabetes mellitus & 2.61 (1.61 to 4.23$)$ & $<0.001$ & 2.07 (1.25 to 3.41$)$ & 0.005 \\
\hline Mean HR > 75 bpm & $1.98(1.11$ to 3.55$)$ & 0.020 & $1.10(0.56$ to 2.17$)$ & 0.783 \\
\hline $\mathrm{SDNN} \leq 70 \mathrm{~ms}$ & 2.01 (1.22 to 3.33$)$ & 0.007 & 1.71 (0.96 to 3.07$)$ & 0.072 \\
\hline QTVI > -0.47 & 2.54 (1.55 to 4.19$)$ & $<0.001$ & $1.12(0.65$ to 1.93$)$ & 0.688 \\
\hline \multirow[t]{2}{*}{$\mathrm{PRD} \geq 5.75 \mathrm{deg}^{2}$} & 4.75 (2.94 to 7.66$)$ & $<0.001$ & $3.03(1.79$ to 5.11$)$ & $<0.001$ \\
\hline & \multicolumn{4}{|c|}{ Cardiovascular Mortality } \\
\hline \multirow[t]{2}{*}{ Risk variable } & \multicolumn{2}{|c|}{ Univariable Cox regression } & \multicolumn{2}{|c|}{ Multivariable Cox regression } \\
\hline & Hazard ratio $(95 \% \mathrm{Cl})$ & $P$ value & Hazard ratio $(95 \% \mathrm{Cl})$ & $P$ value \\
\hline LVEF $\leq 35 \%$ & 4.69 (2.32 to 9.50$)$ & $<0.001$ & $2.71(1.30$ to 5.67$)$ & 0.008 \\
\hline GRACEA $^{A}$ score $\geq 120$ & $5.82(2.75$ to 12.33$)$ & $<0.001$ & 3.80 (1.73 to 8.35$)$ & $<0.001$ \\
\hline Diabetes mellitus & $2.72(1.40$ to 5.31$)$ & 0.003 & $2.16(1.08$ to 4.31$)$ & 0.029 \\
\hline Mean HR > 75 bpm & 2.22 (1.02 to 4.86$)$ & 0.046 & $1.36(0.55$ to 3.38$)$ & 0.510 \\
\hline $\mathrm{SDNN} \leq 70 \mathrm{~ms}$ & $1.89(0.93$ to 3.82$)$ & 0.080 & $1.48(0.65$ to 3.36$)$ & 0.350 \\
\hline QTVI > -0.47 & 1.99 (1.02 to 3.88$)$ & 0.044 & 0.81 (0.39 to 1.70$)$ & 0.586 \\
\hline $\mathrm{PRD} \geq 5.75 \mathrm{deg}^{2}$ & 4.50 (2.33 to 8.69$)$ & $<0.001$ & 2.99 (1.45 to 6.17$)$ & 0.003 \\
\hline
\end{tabular}

1B and Table 1). Patients in the post-MI cohort were enrolled between May 2000 and March 2005 at 2 university centers in Munich, Germany: the German Heart Centre and the Klinikum Rechts der Isar $(21,22)$. Eligible patients had survived acute MI ( $<4$ weeks), were aged 80 years or more, had sinus rhythm, and did not meet the criteria for secondary prophylactic implantation of ICD before hospital discharge. Patients in the stress-test cohort were included between October 2001 and December 2008 at the Tampere University Hospital (Finnish Cardiovascular Study) (25). Eligible patients were aged $30-80$ years, were in sinus rhythm, and underwent a clinically indicated exercise test.

Procedures. We performed EP studies according to the hospital's standard operating procedures. No study-specific invasive procedures were performed on any patient. We did not sedate the patients. All EP studies required placement of a pacing electrode in the right atrium. Atrial pacing was performed at a fixed cycle length (CL), below sinus rhythm CL and slightly above Wenckebach CL. In the atrial-pacing cohort, we compared a 5-minute recording during undisturbed sinus rhythm to a 5-minute recording during fixed atrial stimulation. In the adrenergic-blockade cohort, we compared 5-minute tracings before and after i.v. administration of $0.1 \mathrm{mg} / \mathrm{kg}$ metoprolol. Fixed atrial pacing during the entire procedure was used to ensure constant heart rate.

Subjects in the adrenergic-activation cohort were not allowed to eat or drink coffee for 12 hours before the tests. Vigorous exercise and alcohol were forbidden for 48 hours before the tests. All healthy volunteers lay in a supine position in a quiet room for at least 15 minutes before data collection. We used 2 provocations: 2 -minute passive head-up tilt-test at $45^{\circ}$ and 5-minute low-intensity exercise using a bicycle ergometer. For the latter test, the individual workload was set to achieve a constant heart rate of $110 \mathrm{bpm}$. For all physiological studies, we used high-resolution $(2,048 \mathrm{~Hz})$ digital ECG (TMS; Porti System) recorded in Frank leads configuration throughout the entire procedure.
For the post-MI cohort, we used 30-minute high-resolution $(1,600 \mathrm{~Hz})$ digital ECG (TMS; Porti System) recorded in Frank leads configuration. Recordings were performed within the second week after MI in resting conditions in the morning hours and in a supine position. We additionally performed a 24-hour Holter recording (Oxford Excel Holter System, Oxford Instruments; Pathfinder700, Reynolds Medical; and Mortara Holter System, Mortara Instrument) within the second week after MI. For the stress-test cohort, an upright bicycle was used for the exercise test. The workload was increased from 20 to $30 \mathrm{~W}$ in a step-wise manner $(10$ to $30 \mathrm{~W} / \mathrm{min})$. For calculation of PRD, a preexercise period of at least 2.5 minutes was recorded using 12-channel digital ECG $(500 \mathrm{~Hz})$, which was converted into the Frank leads configuration by means of the inverse Dower matrix (39).

Assessment of PRD. The technique used to calculate PRD is illustrated in Figure 2 and in Supplemental Figures 4-6. The prerequisite for computing $d T^{\circ}$ is an ECG tracing recorded in or converted to the 3 orthogonal axes $\mathrm{X}, \mathrm{Y}$, and $\mathrm{Z}$. The time positions of the $\mathrm{T}$-waves were identified using previously published algorithms $(40,41)$. The end of each T-wave was set as the reference point (amplitude $=0 \mathrm{mV}$ ). The first step in calculating the new parameter was to transform the Cartesian coordinates $\mathrm{X}, \mathrm{Y}$, and $\mathrm{Z}$ (Supplemental Figure 4A) into a time series of polar coordinates defined by 2 angles (elevation and azimuth) and the resultant-force amplitude XYZ (Supplemental Figure 4B). For example, we selected a time point $t 1$ (Supplemental Figure 4B) and decomposed the XYZ vector into 2 orthogonal vectors on the $y$ axis and the transverse (XZ) plane. The angle between the vector and the $y$ axis was termed the elevation (42) (Supplemental Figure $4 \mathrm{D})$, with an angle of $0^{\circ}$ defined as the vector pointing to the caudal direction. The angle between the vector on the transverse plane and the $x$ axis was termed the azimuth (42).

On the basis of the 3 new time signals of the polar coordinates, we defined the weight-averaged direction of repolarization, which can be 


\begin{abstract}
Table 3
Multivariable association of TWA and PRD with all-cause and cardiovascular mortality in 2,965 patients undergoing a clinically indicated exercise test (stress-test cohort)
\end{abstract}

Risk variable
Age, continuous
Sex (yes, no)
Diabetes mellitus (yes, no)
Previous Ml (yes, no)
Beta blocker (yes, no)
TWA, continuous
PRD, continuous
TWA x PRD

Risk variable

Age, continuous

Sex (yes, no)

Previous MI (yes, no)

Beta blocker (yes, no)

TWA $\times$ PRD
Multivariable Cox regression analysis

\begin{tabular}{cccr}
\multicolumn{2}{c}{ All-cause mortality } & \multicolumn{2}{c}{ Cardiovascular mortality } \\
Beta (95\% CI) & $\boldsymbol{P}$ value & Beta $(\mathbf{9 5 \%}$ CI) & $\boldsymbol{P \text { value }}$ \\
$0.055(0.042$ to 0.068$)$ & $<0.001$ & $0.044(0.027$ to 0.063$)$ & $<0.001$ \\
$-0.535(-0.790$ to -0.280$)$ & $<0.001$ & $-0.960(-1.386$ to -0.531$)$ & $<0.001$ \\
$-0.393(-0.675$ to -0.111$)$ & 0.006 & $-0.291(-0.721$ to 0.139$)$ & 0.184 \\
$0.205(-0.049$ to 0.459$)$ & 0.115 & $0.288(-0.080$ to 0.656$)$ & 0.126 \\
$0.350(0.085$ to 0.614$)$ & 0.010 & $0.582(0.164$ to 0.999$)$ & 0.006 \\
$0.175(0.007$ to 0.284$)$ & 0.002 & $0.274(0.133$ to 0.414$)$ & $<0.001$ \\
$0.198(0.103$ to 0.292$)$ & $<0.001$ & $0.269(0.136$ to 0.401$)$ & $<0.001$ \\
$-0.091(-0.160$ to -0.022$)$ & 0.010 & $-0.136(-0.244$ to -0.048$)$ & 0.002
\end{tabular}

Beta, standardized coefficients.

described by a set of 2 polar coordinates that we called the weight-averaged azimuth (WAA) and the weight-averaged elevation (WAE). WAA and WAE can be calculated using Equations 1 and 2, respectively. For each time point $t$, the resultant-force amplitude XYZ, represented as $A m p(t)$ in Equations 1 and 2, was multiplied by the corresponding values for azimuth and elevation at the same time point. The $(A m p(t) \times A n g l e(t))$ products were initially summed for the entire duration of the T-wave and thereafter divided by the sum of all resultant-force amplitudes. The result of each equation represents the "with-the-amplitude weighted" average angle, which is measured by means of the same units (deg rad) as the angle in Equations 1 and 2. Using the repolarization wave of Supplemental Figure 4 as a backbone, exemplary WAA and WAE values were calculated as illustrated in Supplemental Figure 5.

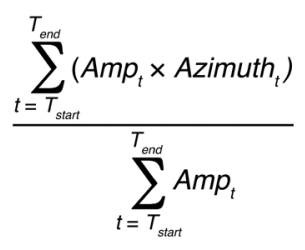

(Equation 1)

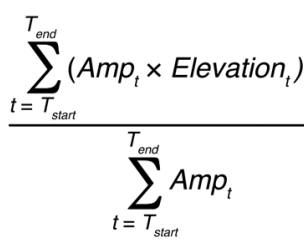

(Equation 2)

We used the angle $d T^{\circ}$ between successive repolarization vectors as an estimate of the instantaneous degree of repolarization instability (Figure $2, \mathrm{~A}-\mathrm{C}$ ). The angle $d T^{\circ}$ was calculated using the dot product (scalar product) equation (43), which by 2 vectors of the same length $r$ can be simplified to Equation 3 as illustrated in Supplemental Figure 6. The $d T^{\circ}$ signal was linearly interpolated with a sampling rate of $2 \mathrm{~Hz}$ and filtered using a low-pass filter to remove artifacts. In order to quantify the periodic components of $d T^{\circ}$, we employed continuous wavelet transformation (Figure 2D). The continuous wavelet transformation provides wavelet coefficients for each scale at each time point. For each scale, the average wavelet coefficient was computed. Finally, scales were converted to pseudofrequencies using an established algorithm (44). PRD was defined as the average wavelet coefficient in the frequency range of $0.1 \mathrm{~Hz}$ or less (Figure 2E).

$$
\begin{aligned}
d T^{\circ}= & a \cos \left[\sin \left(W A E_{1}\right) \times \cos \left(W A A_{1}\right) \times \sin \left(W A E_{2}\right) \times \cos \left(W A A_{2}\right)\right. \\
& +\cos \left(W A E_{1}\right) \times \cos \left(W A E_{2}\right) \\
& \left.+\sin \left(W A E_{1}\right) \times \sin \left(W A A_{1}\right) \times \sin \left(W A E_{2}\right) \times \sin \left(W A A_{2}\right)\right]
\end{aligned}
$$

Assessment of TWA. We assessed TWA by the time-domain MMA method according to previously established technologies (26) (GE Healthcare version 5.2). In brief, the MMA algorithm separates odd from even beats. The average morphologies of both the odd and even beats were calculated separately and continuously updated by a weighting factor of $1 / 8$ or $1 / 32$ of the difference between the ongoing average and the new incoming beats. The update was calculated for every incoming beat, resulting in continual moving averages of the odd and even beats. This approach makes the MMA suitable for TWA analysis during the period of activity or during periods of fluctuating heart rates (non-steady state periods) (45). In addition, algorithms were incorporated to reduce the influence of noise and artifacts, such as those caused by pedaling and respiration (46). The TWA values were calculated continuously during the entire exercise test, from rest to recovery, using all precordial leads. Finally, the maximum TWA value at heart rates of less than $125 \mathrm{bpm}$ was derived.

Assessment of other risk predictors. We assessed LVEF by echocardiography or angiography. We obtained short-term and 24-hour HRV in time and frequency domains as previously proposed (47). Since the standard deviation of all normal-to-normal intervals (SDNN) provided the strongest prognostic power of all HRV measures, we used SDNN as a marker of HRV. We assessed QTVI from the resting ECGs according to previously published technological methods (24). We calculated the GRACE score, which combines several clinical risk factors, specifically patient age, history of previous MI and congestive heart failure, ST-segment deviation, elevated cardiac enzymes, renal impairment, systolic blood pressure and heart rate upon admission, and percutaneous coronary interventions during the hospital stay (23).

Animal study. Seven female domestic pigs $(60-78 \mathrm{~kg})$ were preanesthetized with propofol $(2 \mathrm{mg} / \mathrm{kg}$ i.v. $)$ and anesthetized with $\alpha$-chloralose $(150 \mathrm{mg} / \mathrm{kg}$ i.v. with supplemental doses of $600 \mathrm{mg}$ in $60 \mathrm{ml}$ saline as required), which has been shown to induce only minimal effects on the cardiac autonomic nervous system (20). Immediately after induction of anesthesia, the trachea was cannulated and the lungs were mechanically ventilated with room air. Constant respiratory frequency and tidal volume were maintained by means of volume-controlled ventilation with a fixed tidal volume $(6 \mathrm{ml} / \mathrm{kg})$ and a fixed respiratory rate. In each ani- 
mal, the respiratory rate was set individually (respiratory rate $12-18$ per minute corresponding to a frequency of $0.20-0.30 \mathrm{~Hz}$ ) to maintain normal end-tidal $\mathrm{CO}_{2}$. Respiratory activity was recorded by a piezoelectric thoracic sensor (48). Two hours after the administration of Propofol, a high-resolution 30 -minute ECG $(2,048 \mathrm{~Hz})$ was recorded in the Frank leads configuration.

Statistics. We present continuous variables as medians with IQRs. Categorical data are presented as proportions. Results are presented as mean values with $95 \%$ CI. Results of physiological and EP studies are presented as the ratio of the value after provocation to the corresponding value before provocation with 95\% CI. Differences in the logarithmic ratios were assessed by means of a paired Wilcoxon signed-rank test. To evaluate the effects of respiratory activity on PRD, we estimated the square coherence function between respiratory and $d T^{\circ}$ signals using the cross-spectral method (49). The coherence function (range 0-1) expresses the linear coupling between 2 signals in the frequency domain (50). A square coherence function greater than 0.5 was considered significant $(19,51,52)$. The end points of both prognostic studies were all-cause and cardiovascular mortality. We used the standardized definition of cardiovascular death $(53,54)$. The median follow-up time in the post-MI cohort was 5 years. PRD was dichotomized at the upper quartile of the study population. For dichotomization of other risk markers, we used established cutoff values of $35 \%$ or less for LVEF (55), -0.47 or more for QTVI (24), greater than 75 for mean heart rate (56), $70 \mathrm{~ms}$ or less for SDNN (56), and 120 or more for the GRACE score (23). We estimated survival curves using the Kaplan-Meier method. Multivariable analyses were implemented by the adaptation of Cox and multinomial logistic regression models. The latter method was used to calculate integrated discrimination improvement (IDI) scores (57). The median follow-up time in the stress-test cohort was 6 years. The prognostic powers of PRD and TWA were tested with univariable and multivariable Cox regression analysis,including age, sex, previous MI, the presence of diabetes mellitus, treatment with beta blockers, and the crossterm between TWA and $\mathrm{PRD}(\mathrm{TWA} \times \mathrm{PRD}) . \mathrm{PRD}$, TWA, and age were normalized by subtraction of their mean value and division by their SD and were included as scalar factors in the multivariable model. Differences were considered statisti- cally significant when the 2 -sided $P$ value was less than 0.05 . All statistical analyses were performed using CRAN R, version 2.15.1.

Study approval. The ethics committees of Tübingen, Tampere, and Munich approved the studies performed in the physiological, stress-test, and post-MI cohorts, respectively. Written informed consent was obtained from each participant. The animal protocol was in accordance with the German guidelines for use of living animals and was approved by the local governmental commission for animal research (K 5/10, Regierungspraesidium Tübingen, Baden-Wuerttemberg, Germany).

\section{Acknowledgments}

K. Rizas was awarded for this work with the ESC 2013 Young Investigator Award in Clinical Science. The study was supported in part by grants from the program "Angewandte klinische Forschung” (AKF) of the University of Tübingen (252-1-0 to A. Bauer). No additional external funding was received for this study. The Autonomic Regulation Trial (post-MI cohort) was supported by Bundesministerium für Bildung, Wissenschaft, Forschung und Technologie (13N/7073/7), the Kommission für Klinische Forschung, and the Deutsche Forschungsgemeinschaft (SFB 386). The Finnish Cardiovascular Study (stress-test cohort) was supported by the Medical Research Fund of Tampere University Hospital (grants 9MO48 and 9N035), the Finnish Cultural Foundation, the Finnish Foundation for Cardiovascular Research, the Emil Aaltonen Foundation, and the Tampere Tuberculosis Foundation. The authors thank the staff of the Department of Clinical Physiology for collecting the exercise test data.

Received for publication October 23, 2013, and accepted in revised form January 16, 2014.

Address correspondence to: Axel Bauer, Medizinische Klinik III, Abteilung für Kardiologie und Herz-Kreislauferkrankungen, Eberhard-Karls-Universität Tübingen, Otfried-Müller-Str. 10, 72076 Tübingen, Germany. Phone: 49.7071.29.82922; Fax: 49.7071.29.4550; E-mail: bauer@thebiosignals.org.
1. Huikuri HV, Castellanos A, Myerburg RJ. Sudden death due to cardiac arrhythmias. N Engl J Med. 2001;345(20):1473-1482.

2. Moss AJ, et al. Prophylactic implantation of a defibrillator in patients with myocardial infarction and reduced ejection fraction. $N$ Engl J Med. 2002;346(12):877-883.

3. Hunt SA, et al. 2009 focused update incorporated into the ACC/AHA 2005 Guidelines for the Diagnosis and Management of Heart Failure in Adults: a report of the American College of Cardiology Foundation/American Heart Association Task Force on Practice Guidelines: developed in collaboration with the International Society for Heart and Lung Transplantation. Circulation. 2009;119(14):e391-e479.

4. Task Force for Diagnosis and Treatment of Acute and Chronic Heart Failure 2008 of European Society of Cardiology, et al. ESC Guidelines for the diagnosis and treatment of acute and chronic heart failure 2008: the Task Force for the Diagnosis and Treatment of Acute and Chronic Heart Failure 2008 of the European Society of Cardiology. Developed in collaboration with the Heart Failure Association of the ESC (HFA) and endorsed by the European Society of Intensive Care Medicine (ESICM). Eur Heart J. 2008;29(19):2388-2442.

5. Buxton AE. Risk stratification for sudden death: do we need anything more than ejection fraction? Card Electrophysiol Rev. 2003;7(4):434-437.
6. Rosenbaum DS, et al. Electrical alternans and vulnerability to ventricular arrhythmias. $N$ Engl J Med. 1994;330(4):235-241.

7. Lown B, Verrier RL. Neural activity and ventricular fibrillation. NEngl J Med. 1976;294(21):1165-1170.

8. Verrier RL, Antzelevitch C. Autonomic aspects of arrhythmogenesis: the enduring and the new. Curr Opin Cardiol. 2004;19(1):2-11.

9. Cao JM, et al. Relationship between regional cardiac hyperinnervation and ventricular arrhythmia. Circulation. 2000;101(16):1960-1969.

10. Rubart M, Zipes DP. Mechanisms of sudden cardiac death. J Clin Invest. 2005;115(9):2305-2315.

11. Han J, Garcia de Jalon P, Moe GK. Adrenergic effects on ventricular vulnerability. Circ Res. 1964; 14:516-524.

12. Maling HM, Moran NC. Ventricular arrhythmias induced by sympathomimetic amines in unanesthetized dogs following coronary artery occlusion. Circ Res. 1957;5(4):409-413.

13. Kliks BR, Burgess MJ, Abildskov JA. Influence of sympathetic tone on ventricular fibrillation threshold during experimental coronary occlusion. Am J Cardiol. 1975;36(1):45-49.

14. Butrous GS, Gough WB, Restivo M, Yang H, elSherif N. Adrenergic effects on reentrant ventricular rhythms in subacute myocardial infarction. Circulation. 1992;86(1):247-254.

15. Pagani M, et al. Relationship between spectral components of cardiovascular variabilities and direct measures of muscle sympathetic nerve activity in humans. Circulation. 1997;95(6):1441-1448.

16. Malliani A, Pagani M, Lombardi F, Cerutti S. Cardiovascular neural regulation explored in the frequency domain. Circulation. 1991;84(2):482-492.

17. Pagani $M$, et al. Power spectral analysis of heart rate and arterial pressure variabilities as a marker of sympatho-vagal interaction in man and conscious dog. Circ Res. 1986;59(2):178-193.

18. Montano N, et al. Spectral analysis of sympathetic discharge, R-R interval and systolic arterial pressure in decerebrate cats. J Auton Nerv Syst. 1992;40(1):21-31.

19. Furlan R, et al. Oscillatory patterns in sympathetic neural discharge and cardiovascular variables during orthostatic stimulus. Circulation. 2000;101(8):886-892.

20. Nearing BD, Verrier RL. Tracking cardiac electrical instability by computing interlead heterogeneity of T-wave morphology. J Appl Physiol. 2003;95(6):2265-2272.

21. Barthel P, et al. Spontaneous baroreflex sensitivity: Prospective validation trial of a novel technique in survivors of acute myocardial infarction [published online ahead of print: April 16, 2012]. Heart Rhythm.doi:10.1016/j.hrthm.2012.04.017.

22. Barthel $P$, et al. Respiratory rate predicts outcome after acute myocardial infarction: a prospective cohort study [published online ahead of print: December 14, 2012]. Eur Heart J. doi:10.1093/ 
eurheartj/ehs 420 .

23. Granger CB, et al. Predictors of hospital mortality in the global registry of acute coronary events. Arch Intern Med. 2003;163(19):2345-2353.

24. Piccirillo G, et al. QT variability strongly predicts sudden cardiac death in asymptomatic subjects with mild or moderate left ventricular systolic dysfunction: a prospective study. Eur HeartJ. 2007; 28(11):1344-1350.

25. Nieminen T, et al. T-wave alternans predicts mortality in a population undergoing a clinically indicated exercise test. Eur Heart J. 2007;28(19):2332-2337.

26. Nearing BD, Verrier RL. Modified moving average analysis of T-wave alternans to predict ventricular fibrillation with high accuracy. J Appl Physiol. 2002;92(2):541-549.

27. Slawnych MP, et al. Post-exercise assessment of cardiac repolarization alternans in patients with coronary artery disease using the modified moving average method. J Am Coll Cardiol. 2009;53(13):1130-1137.

28. Verrier RL, Kumar K, Nearing BD. Basis for sudden cardiac death prediction by T-wave alternans from an integrative physiology perspective. Heart Rhythm. 2009;6(3):416-422.

29. Tanabe Y, et al. Sympathetic stimulation produces a greater increase in both transmural and spatial dispersion of repolarization in LQT1 than LQT2 forms of congenital long QT syndrome. J Am Coll Cardiol. 2001;37(3):911-919.

30. Antzelevitch C. Transmural dispersion of repolarization and the T wave. Cardiovasc Res. 2001; 50(3):426-431.

31. Shimizu W, Antzelevitch C. Cellular basis for the ECG features of the LQT1 form of the long-QT syndrome: effects of beta-adrenergic agonists and antagonists and sodium channel blockers on transmural dispersion of repolarization and torsade de pointes. Circulation. 1998;98(21):2314-2322.

32. Vaseghi M, Shivkumar K. The role of the autonomic nervous system in sudden cardiac death. Prog Cardiovasc Dis. 2008;50(6):404-419.

33. Wei K, Dorian P, Newman D, Langer A. Association between QT dispersion and autonomic dysfunction in patients with diabetes mellitus. J Am Coll Cardiol. 1995;26(4):859-863.
34. Narayan SM, Bayer JD, Lalani G, Trayanova NA. Action potential dynamics explain arrhythmic vulnerability in human heart failure: a clinical and modeling study implicating abnormal calcium handling.J Am Coll Cardiol. 2008;52(22):1782-1792.

35. Nearing BD, Huang AH, Verrier RL. Dynamic tracking of cardiac vulnerability by complex demodulation of the T wave. Science. 1991;252(5004):437-440.

36. Nieminen T, et al. The Finnish Cardiovascular Study (FINCAVAS): characterising patients with high risk of cardiovascular morbidity and mortality. BMC Cardiovasc Disord. 2006;6:9.

37. Verrier RL, et al. Microvolt T-wave alternans physiological basis, methods of measurement, and clinical utility--consensus guideline by International Society for Holter and Noninvasive Electrocardiology. J Am Coll Cardiol. 2011;58(13):1309-1324.

38. Tanno K, et al. Microvolt T-wave alternans as a predictor of ventricular tachyarrhythmias: a prospective study using atrial pacing. Circulation. 2004; 109(15):1854-1858.

39. Edenbrandt L, Pahlm O. Vectorcardiogram synthesized from a 12-lead ECG: superiority of the inverse Dower matrix. J Electrocardiol. 1988;21(4):361-367.

40. Laguna P, Jané R, Caminal P. Automatic detection of wave boundaries in multilead ECG signals: validation with the CSE database. Comput Biomed Res. 1994;27(1):45-60.

41. Pan J, Tompkins WJ. A real-time QRS detection algorithm. IEEE Trans Biomed Eng. 1985;32(3):230-236.

42. Shvilkin A, et al. Vectorcardiographic determinants of cardiac memory during normal ventricular activation and continuous ventricular pacing. Heart Rhythm. 2009;6(7):943-948

43. Lipschutz S, Spiegel M, Spellman D. Schaum's Outline of Vector Analysis. 2nd ed. McGraw-Hill Professional; 2009.

44. Abry P. Ondelettes et turbulences: Multirésolutions, algorithmes de décomposition, invariance d'échelle et signaux de pression. Paris, France: Diderot Editeur Arts Sciences, 1997.

45. Hostetler B, et al. Detect short run of TWA event with time-domain algorithm. Compute Cardiol. 2005;2005:483-486.

46. Kaiser W, Findeis M, Young BJ. Improving T-wave alternans measurement quality by reducing noise and artifacts. Compute Cardiol. 2004;2004:445-448.

47. Malik M, et al. Heart rate variability. Standards of measurement, physiological interpretation, and clinical use. Task Force of the European Society of Cardiology and the North American Society of Pacing and Electrophysiology. Eur Heart J. 1996; 17(3):354-381.

48. Aminian K, Thouvenin X, Robert P, Seydoux J, Girardier L. A piezoelectric belt for cardiac pulse and respiration measurements on small mammals. IEEE Engineering in Medicine and Biology Society, 1992 14th Annual International Conference of the IEEE. 7:2663-2664.

49. Saul JP, et al. Transfer function analysis of the circulation: unique insights into cardiovascular regulation. Am J Physiol. 1991;261(4 pt 2):H1231-H1245.

50. Robbe HW, et al. Assessment of baroreceptor reflex sensitivity by means of spectral analysis. Hypertension. 1987;10(5):538-543.

51. Song J-G, et al. Effects of bilateral stellate ganglion block on autonomic cardiovascular regulation. Circ J. 2009;73(10):1909-1913.

52. Maslyukov PM, Nozdrachev AD. Rhythmic electrical activity in branches of the stellate ganglion in the cat during postnatal ontogenesis. Neurosci Behav Physiol. 2007;37(5):505-508.

53. Hicks KA, et al. Standardized definitions for cardiovascular and stroke end point events in clinical trials. CDISC. http://www.cdisc.org/stuff/contentmgr/files/0/2356ae38ac190ab8ca4ae0b222392b37/ misc/cdisc_november_16_2010.pdf. Published October 20, 2010. Accessed February 20, 2014.

54. Cutlip DE, et al. Clinical end points in coronary stent trials: a case for standardized definitions. Circulation. 2007;115(17):2344-2351.

55. Bardy GH, et al. Amiodarone or an implantable cardioverter-defibrillator for congestive heart failure. N Engl J Med. 2005;352(3):225-237.

56. Barthel P, et al. Reflex and tonic autonomic markers for risk stratification in patients with type 2 diabetes surviving acute myocardial infarction. Diabetes Care. 2011;34(8):1833-1837.

57. Van Calster B, Van Huffel S. Integrated discrimination improvement and probability-sensitive AUC variants. Stat Med. 2010;29(2):318-319. 